\title{
CARACTERIZACIÓN FÍSICO QUÍMICA DE LOS COMPONENTES VOLÁTILES DEL Tecoma fulva subsp. arequipensis (cahuato)
}

\author{
Jorge Chávez Fernández*, Virginia Lizárraga Lazo, Eleana Vargas de Nieto
}

\begin{abstract}
RESUMEN
El género Tecoma consta de 14 especies, distribuidas en toda América; crece en zonas tropicales. En la región Arequipa - Perú, existen tres especies, siendo una de ellas la T. fulva subsp. arequipensis, la cual fue recolectada en el distrito de Sogay a 2600 m.s.n.m. de la región Arequipa. Los componentes volátiles fueron extraídos por destilación con arrastre de vapor en agua, el rendimiento fue del $0,40 \%$ en hojas y flores de muestra fresca obteniendo una resina blanca, densidad $0,85 \mathrm{~g} / \mathrm{mL}$, índice de refracción 1,464 a $20^{\circ} \mathrm{C}$ y soluble en etanol; se detectó la presencia de terpenos por cromatografía en capa fina. Por el método de extracción de maceración en diclorometano y éter de petróleo de hojas y flores de muestra seca con 13 $\%$ de humedad residual, se concentró en rotaevaporador obteniendo 5,4 \% de extracto seco, el extracto fue analizado por cromatografía de gases acoplado a un espectro de masas (GC/ $\mathrm{EM})$, se identificó 11 componentes en hojas, siendo el componente mayoritario el terpinen4-ol con 37,45 \% de abundancia, del extracto diclorometánico. En el extracto etéreo de flores se identificaron 18 constituyentes, siendo el componente mayoritario el fitol con 16,38\% de abundancia. En el extracto etanólico de hojas de $T$. fulva subsp. arequipensis purificado por $\mathrm{CC}-\mathrm{CG} / \mathrm{EM}$ en tres fracciones se detectó la presencia de: fracción 1 se identificó $\gamma$-sitosterol con 50,1\% de abundancia, fracción 3 se identificó ácido hexadecanoico con 55,2 $\%$ de abundancia y en la fracción 4 se identificó 1,3-dihidro-3,3-dimetil-2H-indol-2-ona con $36,1 \%$ de abundancia. El espectro infrarrojo con transformada de Fourier (FTIR) confirmó la presencia de: alcoholes, fenoles, ácidos carboxílicos, cetonas $\alpha, \beta$ insaturadas, alquenos, hidrocarburos aromáticos y aminas; es importante resaltar que los componentes detectados e identificados son terpenoides y sesquiterpenoides.
\end{abstract}

Palabras clave: Tecoma fulva subsp. arequipensis, extracto diclorometano, terpenos y sesquiterpenoides, GC-MS, FTIR.

\footnotetext{
Sección Química Orgánica. Departamento Académico de Química. Universidad Nacional de San Agustín de Arequipa. Calle Santa Catalina 117 - Cercado. Arequipa - Perú. jchavezf@unsa.edu.pe
} 


\title{
CHEMICAL PHYSICAL CHARACTERIZATION OF VOLATILE COMPONENTS OF Tecoma fulva subsp. arequipensis (cahuato)
}

\begin{abstract}
The genus Tecoma consists of 14 species, distributed throughout the Americas; grows in tropical areas. In the Arequipa - Peru region, there are three species, one of them being T. fulva subsp. arequipensis, which was collected in the Sogay district at 2600 m.s.n.m. of the Arequipa region. The volatile components were extracted by steam distillation in water, the yield was $0.40 \%$ in fresh sample leaves and flowers, obtaining a whitresin, density $0.85 \mathrm{~g} / \mathrm{mL}$, refractive index 1.464 at $20^{\circ} \mathrm{C}$ and soluble in ethanol; the presence of terpenes was detected by thin layer chromatography. By the extraction method of maceration in dichloromethane and petroleum ether of leaves and flowers of a dry sample with $13 \%$ residual humidity, it was concentrated in a rotary evaporator obtaining $5.4 \%$ of dry extract, the extract was analyzed by gas chromatography coupled to In a mass spectrum (GC/MS), 11 components were identified in leaves, the majority component being terpinen-4-ol with 37.45 $\%$ abundance, of the dichloromethane extract. In the ether extract of flowers, 18 constituents were identified, the main component being phytol with $16.38 \%$ abundance. In the ethanolic extract of leaves of T. fulva subsp. arequipensis purified by CC-GC/ MS in three fractions the presence of was detected: fraction $1 \gamma$-sitosterol was identified with 50.1\% abundance, fraction 3 hexadecanoic acid was identified with $55.2 \%$ abundance and in fraction 4 1,3-dihydro-3,3-dimethyl-2H-indol-2-one was identified with $36.1 \%$ abundance. The Fourier transform infrared spectrum (FTIR) confirmed the presence of: alcohols, phenols, carboxylic acids, $\alpha, \beta$ unsaturated ketones, alkenes, aromatic hydrocarbons and amines; It is important to highlight that the components detected and identified are terpenoids and sesquiterpenoids.
\end{abstract}

Key words: Tecoma fulva subsp. arequipensis, dichloromethane extract, terpenes and sesquiterpenoids, GC-MS, FTIR.

\section{INTRODUCCIÓN}

La familia Bignoniaceae comprende alrededor de 120 géneros y 800 especies distribuidas en regiones tropicales y subtropicales ${ }^{1}$; entre ellos se tiene el género Tecoma, constituyendo 14 especies de arbustos, crece en toda Latinoamérica ${ }^{2-3}$. En el Perú, se encuentra distribuida en los departamentos de Ica, Arequipa, Tacna y Moquegua; desarrollándose entre 2000 a 3000 m.s.n.m. En la región Arequipa - Perú, hay tres especies: T. fulva subsp. arequipensis, $T$. stans y T. sambusifolia; utilizada en medicina tradicional: como agente antimicrobiano para el tratamiento de algunos tipos de cáncer y forraje del ganado ${ }^{4-5}$.

El extracto etanólico de las flores de $T$. stans tiene actividad antidiabética, debido a la presencia de los alcaloides tecomina y tecostamina ${ }^{6}$. La literatura científica reporta, que el género Tecoma contiene compuestos bioactivos, como: saponinas, glicósidos, flavonoides, alcaloides, aceites esenciales, terpenos, fitoesteroles, antraquinonas, taninos, etc..$^{3-4}$. 
El aceite esencial de las hojas de T. smithii Will, obtenido por hidrodestilación presentan actividad antimicrobiana y antioxidante, determinando sus características fisicoquímicas, fueron identificados los metabolitos secundarios por CG/EM ${ }^{1,7-9}$. La especie T. fulva subsp. arequipensis en el Perú, conocida tradicionalmente con el nombre de cahuato o huarango; utilizada en medicina popular para aliviar el dolor estomacal, antiinflamatorio y cicatrización de heridas, además es considerada como planta ornamental, para leña y elaboración de canastas.

El objetivo de la investigación es extraer, separar, purificar e identificar los componentes volátiles orgánicos de T. fulva subsp. arequipensis, obtenidas por dos métodos y su caracterización fitoquímica fue utilizando fundamentalmente técnicas de CG/EM y FTIR, debido a que no existen antecedentes bibliográficos de la especie en estudio.

\section{PARTE EXPERIMENTAL}

\section{Recolección y secado de la muestra}

Las hojas y flores del T. fulva subsp. arequipensis fue recolectada en el distrito de Sogay, región Arequipa a 2600 m.s.n.m. (16 $56^{\prime} 75^{\prime}$ ' sur y $71^{\circ} 43^{\prime} 75^{\prime}$ 'oeste) en los meses de abril y mayo del 2017; la muestra fue secada a temperatura ambiente, $20^{\circ} \mathrm{C}$ por el lapso de siete días. La clasificación taxonómica del vegetal fue realizada en el herbario de UNSA.

\section{Materiales, reactivos y equipos}

Todos los reactivos y solventes fueron de grado analítico, cromatofolios de silicagel $60 \mathrm{~F}_{254}$ de las marcas Merck y Acros. Rotaevaporador marca Bucchi modelo R 300, CG/EM; modelo Shimadzu GC-2010, GCROM versión 21000; GC Detector MS serial Nº 020524800562 AE ROM VERSIÓN 1,02 Modelo Etapa Dual TMP (Ultra) Fase móvil o gas de arrastre: helio, fase estacionaria: silica fundida $5 \%$ y $95 \%$ de fenildimetil polisiloxano. Columna: [GC2010]. Nombre: Rtx-5MS. Espesor: 0,25 $\mu \mathrm{m}$. Longitud: $30 \mathrm{~m}$. Diámetro interior: 0,25 mm. Temperatura máxima utilizable: $330^{\circ} \mathrm{C}$. Inyector: automático.

Refractómetro Abbe. Espectrofotómetro Perkin Elmer Spectrum IR Modelo Frontier versión 10.6.0, en la región del infrarrojo medio comprendida entre 400 y $4000 \mathrm{~cm}^{-1}$. Bomba de vacío de alta presión marca Corning-EEL 852; Vortex marca Labor Muszeripari tipo LE-203.

Para el extracto etanólico se utilizó el CG/EM marca Thermo Scientific Trace ${ }^{\mathrm{TM}}$ serie 1300, Pre corrida tiempo: 10,00 minutos tiempo de equilibrio: 0,50 minutos. Inicio de preparación: 0,00 min. Encendido / apagado del horno: Encendido Habilitación criogénica: Apagado Umbral criogénico: 50,0 ${ }^{\circ} \mathrm{C}$ Tiempo de espera criogénico: 60,00 min Temperatura inicial: $100,0{ }^{\circ} \mathrm{C}$ Tiempo de retención inicial: 3,00 min Número de rampas: 1 rampa 01 velocidad: $20,0^{\circ} \mathrm{C} / \mathrm{min}$ Rampa 01 temperatura final: $300,0^{\circ} \mathrm{C}$ Rampa 01 tiempo de espera: 20,00 min S / SL - Método frontal Modo S / SL: temperatura sin división habilitada: temperatura activada: $220{ }^{\circ} \mathrm{C}$ Habilitación de flujo dividido: Encendido Flujo dividido: 50,0 ml/min Tiempo sin división: 1,50 min Flujo de purga: 5,0 ml/min Purga de tabique constante: En modo 
portador: Flujo constante Habilitación de flujo portador: En portador flujo: 1,000 mL/minutos Compensación de vacío: Activación del ahorrador de gas portador: Flujo del ahorrador de gas portador: 5,0 mL/minuto Tiempo de ahorro de gas portador: 2,00 minutos Activación de retrolavado: Tiempo de inicio de retrolavado: 1,50 minutos Tiempo de ejecución del GC Auxiliar Zonas Método Auxiliar temperatura 1 habilitado: En Auxiliar temperatura 1: $240{ }^{\circ} \mathrm{C}$ Auxiliar temperatura 2 habilitada.

\section{Extracción de los componentes volátiles}

Los componentes volátiles orgánicos de hojas y flores frescas de T. fulva subsp. arequipensis, fueron extraídos por dos métodos: destilación con arrastre de vapor y por maceración en solventes orgánicos. El método de arrastre de vapor se realizó en un equipo tipo Clevenger, la extracción se realizó por triplicado de masa promedio de $180 \mathrm{~g}$, de dos a tres horas. El destilado obtenido, se extrajo con $\mathrm{CH}_{2} \mathrm{Cl}_{2}$ y éter de petróleo, se secó con $\mathrm{Na}_{2} \mathrm{SO}_{4}$ anhidro ${ }^{13}$, para luego identificar los compuestos químicos presentes por CCF. Por maceración se utilizó $25 \mathrm{~g}$ de hojas y flores de muestra seca por duplicado con $250 \mathrm{~mL}$ de solventes, previamente determinando la humedad residual, por el método gravimétrico; los solventes orgánicos empleados fueron: diclorometano, éter de petróleo y etanol. Durante cinco horas diarias con agitación constante con ayuda de un equipo vortex; macerado por cuatro días. El extracto se filtró en papel Whatman $\mathrm{N}^{\circ} 1$, se concentró en rotaevaporador ${ }^{13-14}$.

\section{Caracterización física y separación por CCF}

En el aceite esencial obtenido de la muestra fresca, se realizó la caracterización física: densidad por el método del picnómetro, índice de refracción y la solubilidad en solventes orgánicos, siendo separados sus componentes por CCF en cromatofolios de silicagel, como fase móvil se utilizó acetato de etilo - tolueno (7:93), como agente revelador fue empleado solución de vainillina - $\mathrm{H} 2 \mathrm{SO} 4$, se comparó las moléculas orgánicas presentes en el aceite esencial, del vegetal en estudio con los estándares: $\boldsymbol{\alpha}$-terpineno, p-cimeno, pineno terpinen4-ol, borneol, timol, mentona y mentol ${ }^{15}$.

\section{Identificación y cuantificación de los extractos orgánicos por CG/EM}

Para la identificación de los componentes volátiles de los extractos orgánicos por maceración se concentró en rotaevaporador; con los concentrados orgánicos de hojas y flores obtenidos por separado se masó $0,5 \mathrm{~g}$ de muestra, aforando en fiola de $10 \mathrm{~mL}$ con hexano grado GC. Se depositó en el sonicador por 45 minutos. Filtro el extracto con filtro de jeringa 0,22 $\mu \mathrm{m}$ y colocar directo en el vial de lectura. Para ser analizados en las mismas condiciones por CG/ EM, cada uno de los extractos.

\section{Purificación del extracto etanólico por $\mathrm{CC}$ y análisis por CG/EM}

El fraccionamiento del extracto etanólico de las hojas y flores secas del T. fulva subsp. arequipensis se realizó por cromatografía en columna (CC) abierta, usando como fase estacionaria silicagel G-200, como fase móvil hexano-acetato de etilo, en diferentes proporciones $(9: 1 ; 8: 2)$; fueron separadas las fracciones 1,3 y 4 , concentradas en rotaevaporador para luego determinar e identificar los componentes presentes por CG/EM. Las condiciones de análisis en $200 \mu \mathrm{L}$ de cada fracción fueron cargados en sendos insertos 
para cada vial del autoinyector, la aguja es lavada con una mezcla de metanol-acetato de etilo, antes y después de cada inyección alrededor de 10 veces en total, se carga la jeringa para ser inyectada cuidadosamente, en el equipo Thermo Scientific Trace ${ }^{\mathrm{TM}}$ serie 1300 CG/EM.

El equipo GC se encuentra a una temperatura inicial de $60^{\circ} \mathrm{C}$ en el horno, para operar en split, 1/50; reteniendo ese valor por 3 minutos luego se inicia una rampa de $20 \mathrm{Grd} / \mathrm{min}$. hasta $300^{\circ} \mathrm{C}$ en el horno de 10 a $15 \mathrm{~min}$, para limpiar la columna capilar. El inyector del GC se estableció a $220^{\circ} \mathrm{C}$, y la línea de transferencia del GC al MS a $240^{\circ} \mathrm{C}$. La fuente de iones está a $200^{\circ} \mathrm{C}$ y se opera a $70 \mathrm{eV}$, barriendo de los 40 a los $50 \mathrm{Da}$. El gas carrier es helio de alta pureza, el mismo que se encuentra presente dentro del EM, la columna capilar es una OV-5, para EM, de fase químicamente unida y entrelazada al capilar de la misma.

Estos ensayos se realizaron en el Instituto de Investigaciones en Tecnología Química (INTEQUI) de la Universidad Nacional de San Luis Argentina.

\section{Análisis por Espectroscopia FT-IR}

Las hojas y flores secas de T. fulva subps. arequipensis fueron maceradas en diclorometano por el lapso de tres días con agitación constante, se filtró a vacío haciendo uso de papel filtro Whatman $\mathrm{N}^{\circ} 1$, el extracto obtenido se concentró en rotaevaporador hasta polvo seco, la muestra se re disolvió en diclorometano; el análisis espectroscópico se realizó utilizando FTIR, en porcentaje de transmitancia que fue útil para detectar los picos característicos y los grupos funcionales presentes en los extractos purificados, los mismos que fueron registrados e interpretados con tablas espectroscópicas.

\section{RESULTADOS Y DISCUSIÓN}

El rendimiento del aceite esencial por arrastre con vapor de agua de hojas y flores frescas de T. fulva subsp. arequipensis, fue de $0,40 \%$, de aspecto resinoso de color blanco, siendo inestable y soluble en agua luego de la extracción; por lo que no se aplicó la técnica de GC/ $\mathrm{EM}$, de densidad $0,85 \mathrm{~g} / \mathrm{mL}$ e índice de refracción 1,464 a $20^{\circ} \mathrm{C}$, soluble en etanol absoluto, éter de petróleo y diclorometano.

Para el vegetal con $13 \%$ de humedad residual, se utilizó el método de maceración en solventes orgánicos por cuatro días y concentrado en rotaevaporador, se obtuvieron los siguientes rendimientos: extracto en diclorometano 5,4 \%; extracto en éter de petróleo 5,0 \% y el extracto en etanol al $98 \%$ fue de $6,2 \%{ }^{15}$.

Por CCF de los componentes volátiles de T. fulva subsp. arequipensis, al ser soluble en agua la resina obtenida se extrajeron los componentes orgánicos en diclorometano y éter de petróleo, de forma cualitativa se pudo identificar, cuatro probables estructuras orgánicas terpenicas, el terpinen-4-ol, $p$-cimeno, mentona y $\alpha$ - pineno (figuras 1 y 2). Los resultados indican que las estructura presentan el factor de retención (RF) similares a los estándares. 


\section{Donde:}

M1: extracto en diclorometano de hojas.

M2: extracto en diclorometano de flores.

\section{Estándares cromatográficos:}

$\alpha$-terpineno $(\alpha \mathrm{T})$, borneol (B), p-cimeno (PC), $\alpha$-pineno $(\alpha \mathrm{P})$, timol (T), terpinen 4-ol (T-ol), mentona (M-CO) mentol (M)

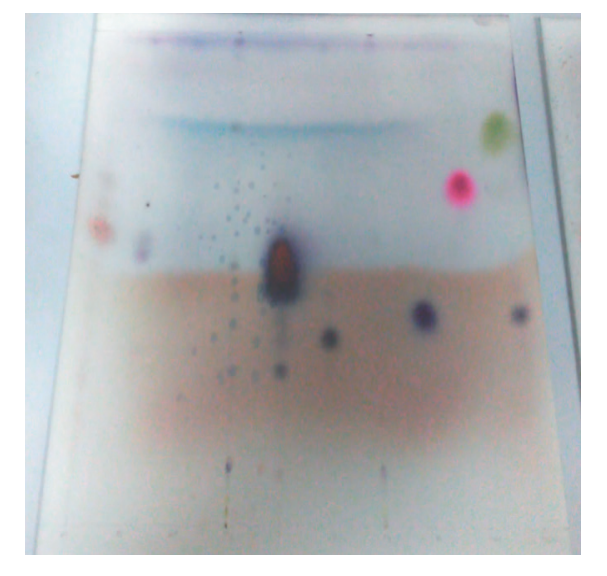

$\alpha \mathrm{T} \quad \mathrm{PC} \quad \alpha \mathrm{P} \quad \mathrm{M} 1 \quad \mathrm{~T} \quad \mathrm{~B} \quad \mathrm{M} 2 \mathrm{M}$ T-ol MCO

Figura 1. Cromatograma del extracto de diclorometano de hojas y flores del T. fulva subsp. arequipensis.

\section{Donde:}

M3: Extracto en éter de petróleo de T. fulva subsp. arequipensis hojas.

M4: extracto en éter de petróleo de T. fulva subsp. arequipensis flores.

Estándares cromatográficos:

$\alpha$-terpineno $(\alpha \mathrm{T})$, borneol (B), p-cimeno (PC), $\alpha$-pineno $(\alpha \mathrm{P})$, timol (T), terpinen 4-ol (T-ol), mentona (M-CO), mentol (M).

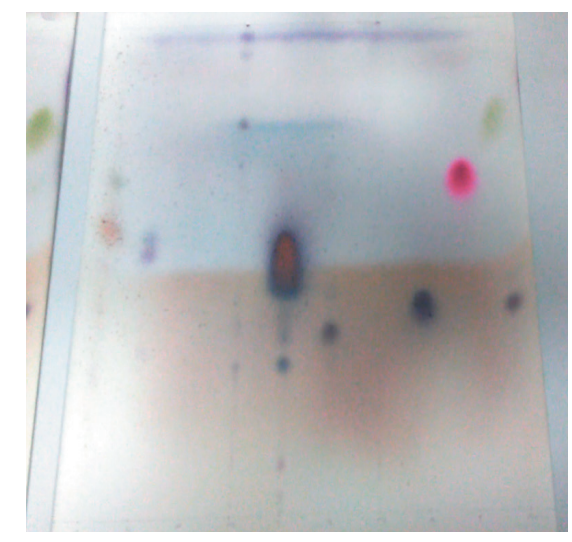

$\alpha \mathrm{T} \quad \mathrm{PC} \alpha \mathrm{P} \quad \mathrm{M} 3 \quad \mathrm{~T} \quad \mathrm{~B} \quad \mathrm{M} 4 \quad \mathrm{M}$ T-ol $\mathrm{MCO}$

Figura 2. Cromatograma del extracto etéreo de hojas y flores del T. fulva subsp. arequipensis. 
Analizando las figuras 1 y 2, se observa que hay presencia de moléculas terpénicas, siendo ello coincidente con estudios realizados en aceites esenciales de otras especies vegetales reportando estructuras terpénicas y terpenoides ${ }^{16-17}$.

Por la CG/EM del extracto en diclorometano de las hojas fueron identificados 11 compuestos orgánicos (tabla 1 y figura 3), correspondiendo a ocho estructuras de naturaleza terpénica, como el 4-terpineol con $37,45 \%$ de abundancia (tabla 1 , figura 3 )

Tabla 1. Composición química del extracto en diclorometano de las hojas de Tecoma fulva subsp. arequipensis.

\begin{tabular}{clc}
\hline $\mathbf{N}^{\circ}$ & \multicolumn{1}{c}{ Compuestos químicos } & Porcentaje $\%$ \\
\hline 1 & oct-1-en-3-ol & 1,42 \\
2 & terpinen-4-ol & 37,45 \\
3 & 2-metilbutanoato de cis hex-3-enil & 1,47 \\
4 & 3,7-dimetilocta-2,6-dieno & 1,95 \\
5 & (1-prop-1-enil)-2-metoxifenol & 13,14 \\
6 & octahidropentalen-1-ol & 10,28 \\
7 & mentol & 5,15 \\
8 & 2,5-diciclopropilpenta-2-en-4-ino & 3,09 \\
9 & (10Z)-pentadec-10-en-1-ol & 8,67 \\
10 & germacreno & 1,44 \\
11 & $\alpha-p i n e n o$ & 7,22 \\
\hline
\end{tabular}

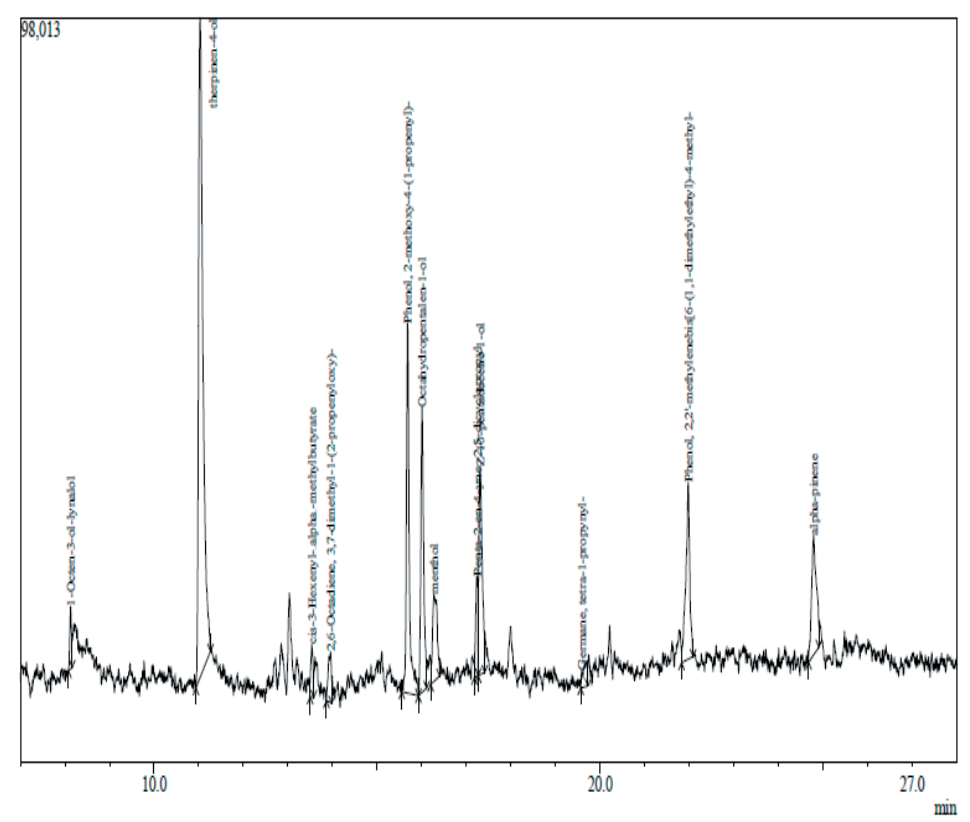

Figura 3. Cromatograma del extracto de hojas de Tecoma fulva subsp. arequipensis en diclorometano por GC - MS 
En el extracto etéreo de flores se detectó la presencia de 18 compuestos orgánicos, correspondiendo a 13 estructuras de naturaleza terpénica, siendo el compuesto mayoritario fitol con 16,38 \% de abundancia (tabla 2, figura 4).

Tabla 2. Composición química del extracto en éter de petróleo de las flores del Tecoma fulva subsp. arequipensis.

\begin{tabular}{clc}
\hline $\mathbf{N}^{\circ}$ & \multicolumn{1}{c}{ Compuestos químicos } & Porcentaje \% \\
\hline 1 & 2-propilpenten-1-ol & 5,0 \\
2 & 3,7-dimetilocta-1,6-dien-3-ol & 2,97 \\
3 & nonanal & 3,16 \\
4 & 4,5-dimetilundec-2-eno & 2,88 \\
5 & 1(3-metilbutil)ciclopenteno & 4,74 \\
6 & 2,6,10-trimetildodecano & 4,30 \\
7 & 2,6,11-trimetildodecano & 4,14 \\
8 & pentadecano & 2,99 \\
9 & (3E)pentadec-3-en-2-ol & 2,97 \\
10 & pentadecanal & 10,48 \\
11 & decan-2-ona & 7,25 \\
12 & 3,7,11,15-tetrametilhexadec-2-en-1-ol & 6,01 \\
13 & (2Z,5Z)pentadeca-2,5-dien-1-ol & 5,52 \\
14 & decano & 6,69 \\
15 & dodecanoato de-1-metiletilo & 3,06 \\
16 & 2-metilpentadecano & 4,31 \\
17 & fitol & 16,38 \\
18 & 2,7,10-trimetildodecano & 3,05 \\
\end{tabular}

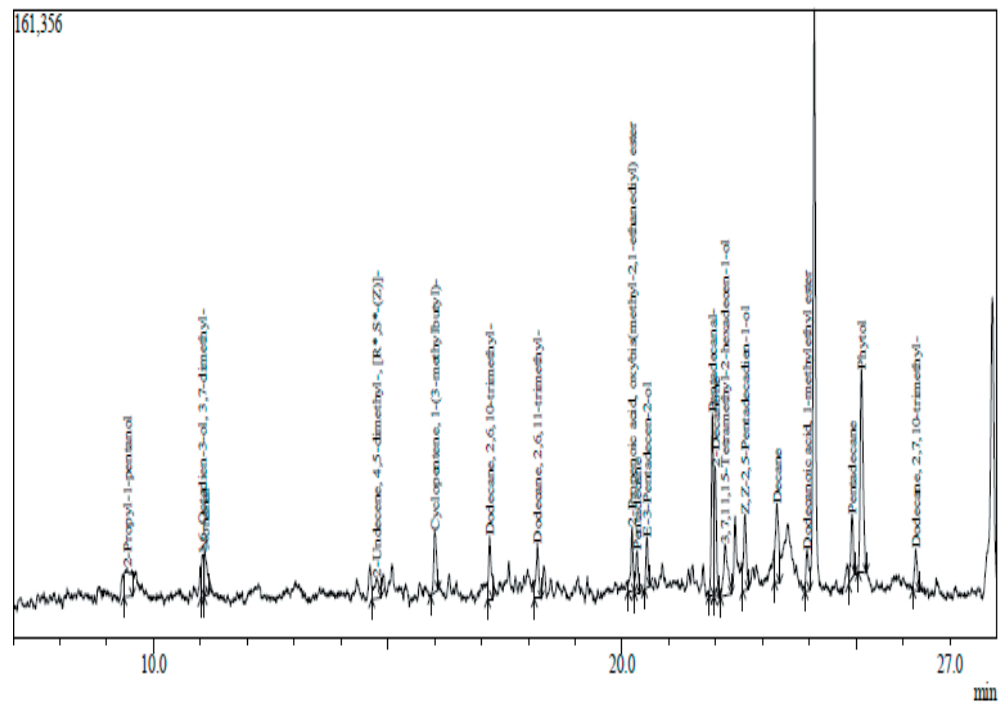

Figura 4. Cromatograma del extracto de flores del Tecoma fulva subsp. arequipensis en éter de petróleo por GC-MS 
El estudio fitoquímico de otras especies del género, como T. smithii, el aceite esencial de hojas frescas presentó 23 constituyentes químicos siendo el fitol el compuesto mayoritario (Kamilia et al. 2016); el extracto etanólico del T. stans se detectó la presencia de 17 constituyentes químicos, siendo estructuras terpénicas con marcada actividad biológica ${ }^{2,18}$.

Del fraccionamiento por cromatografía en columna y CG/EM; en la fracción 1 obtenida del extracto etanólico de las hojas de T. fulva subsp. arequipensis fueron detectados la presencia de 33 constituyentes orgánicos, el esteroide $\gamma$-sitosterol, de fórmula molecular $\mathrm{C}_{29} \mathrm{H}_{50} \mathrm{O}$ y peso molecular $414 \mathrm{~g} / \mathrm{mol}$; fue el de mayor abundancia: (50,1 \%), el espectro de masas puede ser observado en la figura 5. En la fracción 3 se detectaron la presencia de 33 componentes químicos siendo el ácido hexadecanoico con 52,2 \% de mayor abundancia, de fórmula molecular $\mathrm{C}_{16} \mathrm{H}_{32} \mathrm{O}_{2}$ y peso molecular $256 \mathrm{~g} / \mathrm{mol}$, cuyo espectro de masas presentamos en la figura 7. En la fracción 4 se identificaron la presencia de 33 componentes químicos, siendo el 1,3-dihidro-3,3-dimetil-2H-indol-2-ona con 36,1\% el de mayor abundancia, de fórmula molecular $\mathrm{C}_{10} \mathrm{H}_{11} \mathrm{NO}$ y peso molecular $161 \mathrm{~g} / \mathrm{mol}$ lo que se observa en la figura 8 .

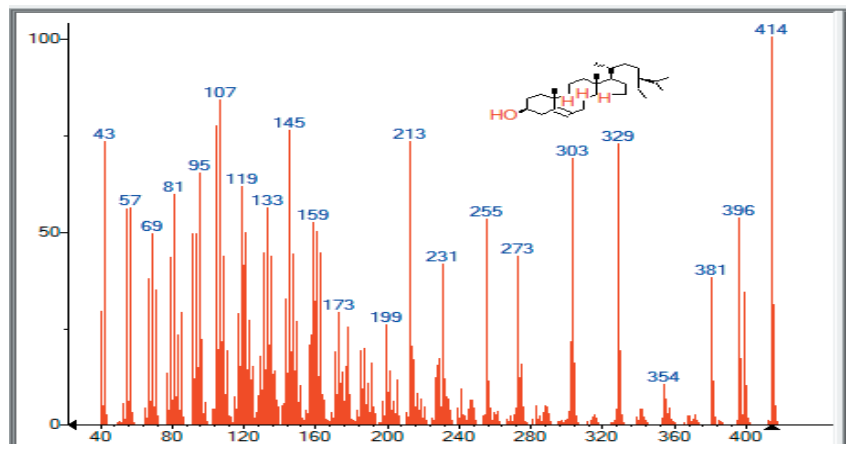

Figura 5. Espectro de masas del $\gamma$-sitosterol en la fracción 1, del extracto en etanol de hojas del Tecoma fulva subsp. arequipensis.

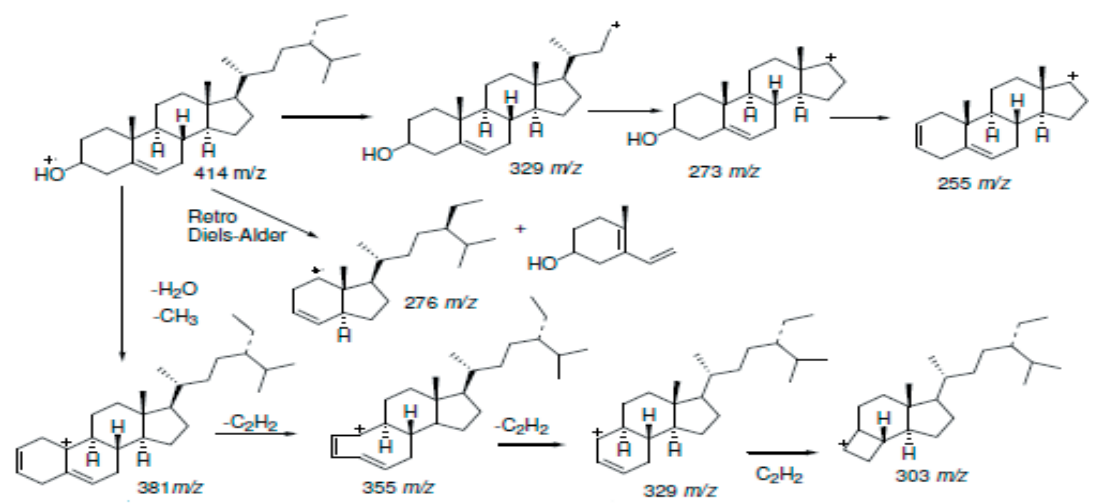

Figura 6. Fragmentación propuesta para el $\gamma$-sitosterol (cortesia de Peniche H. pag. 58, 2016. 
En la figura 6 se observa que a partir de la formación del ión molecular $414 \mathrm{~m} / \mathrm{z}$, se genera la fragmentación típica del $\gamma$-sitosterol con una insaturación $\Delta 5$, los fragmentos $329 \mathrm{~m} / \mathrm{z} \mathrm{y}$ $303 \mathrm{~m} / \mathrm{z}$, además de la pérdida de metilos, el -OH de la posición C-3 y carbonos de la cadena lateral; a través de pérdidas consecutivas de etino $\left(\mathrm{C}_{2} \mathrm{H}_{2}\right)^{19}$.

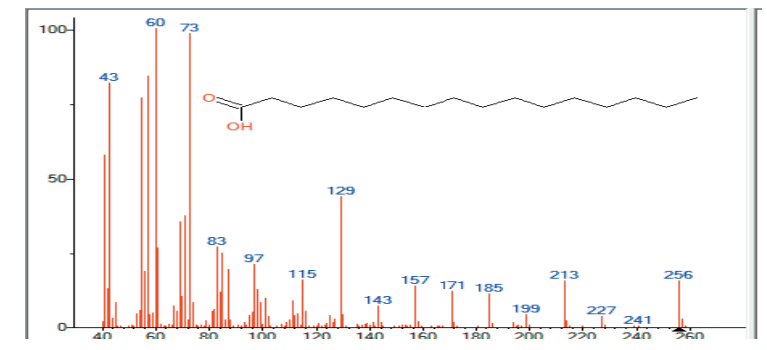

Figura 7. Espectro de masas del ácido hexadecanoico en la fracción 3, del extracto en etanol de hojas del Tecoma fulva subsp. arequipensis.

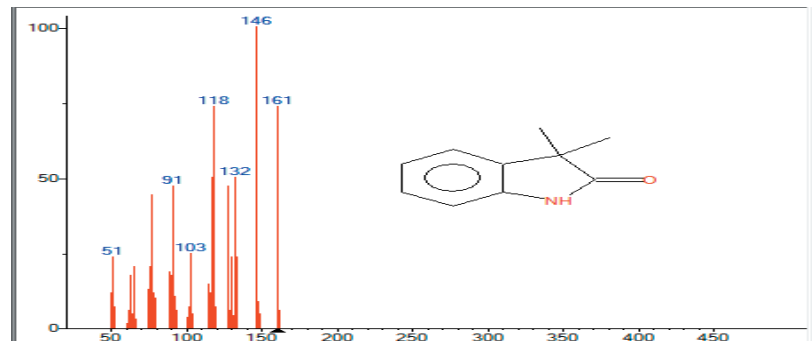

Figura 8. Espectro de masas del 1,3-dihidro-3,3-dimetil-2H-indol -2-ona en la fracción 4, del extracto en etanol de hojas del Tecoma fulva subsp. arequipensis.

La identificación de los componentes químicos en las fracciones fue realizada por comparación con la base de datos de los espectros de masas de la biblioteca científica. Mass Spectral Libraries (NIST 17 and Wiley Libraries) 2014.

Los resultados de los espectros FTIR, los picos detectados y las funciones orgánicas correspondientes al extracto de diclorometano de flores se muestran en la figura 9 y tabla 3 y de las hojas se muestran en la figura $10 \mathrm{y}$ tabla 4 de T. fulva subsp. arequipensis. 


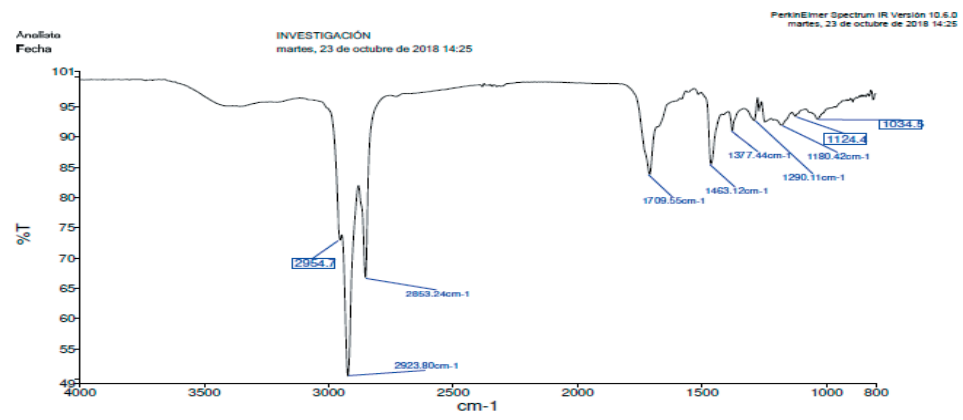

Figura 9. Espectro FTIR del extracto en diclorometano de flores de Tecoma fulva subsp. arequipensis.

Tabla 3. Espectro FTIR del extracto en diclorometano de flores de Tecoma fulva subsp. arequipensis.

\begin{tabular}{cccc}
\hline $\begin{array}{c}\mathbf{N}^{\circ} \\
\text { pico }\end{array}$ & $\ddot{\boldsymbol{v} \mathbf{c m}^{-1}}$ & $\begin{array}{c}\text { Vibración del } \\
\text { grupo funcional }\end{array}$ & Función orgánica \\
\hline $\mathrm{A}$ & 3500 & $\mathrm{O}-\mathrm{H}, \mathrm{N}-\mathrm{H}$ & alcoholes, fenoles, ácidos y aminas \\
$\mathrm{B}$ & 2923 & $\mathrm{C}-\mathrm{H}$ & hidrocarburos saturados: alcanos \\
$\mathrm{C}$ & 2853 & $\mathrm{C}-\mathrm{H}$ & alcanos \\
$\mathrm{D}$ & 1709 & $\mathrm{C}=\mathrm{O}$ & cetonas $\alpha, \beta$ insaturadas, ácidos, ésteres \\
$\mathrm{E}$ & 1463 & $-\mathrm{CH}_{2}-$ & metilenos de alcanos \\
$\mathrm{F}$ & 1377 & $-\mathrm{CH}_{3}$ & metilos de alcanos \\
$\mathrm{G}$ & $1290-1034$ & $\mathrm{C}-\mathrm{C}, \mathrm{C}-\mathrm{O}, \mathrm{C}-\mathrm{N}$ & huella dactilar: alcanos, éteres, aminas \\
\hline
\end{tabular}

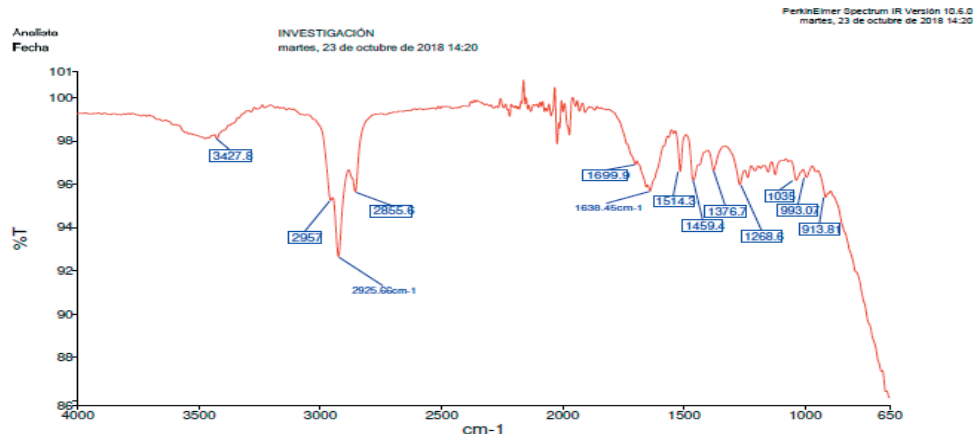

Figura 10. Espectro FTIR del extracto en diclorometano de hojas de Tecoma fulva subsp. arequipensis. 
Tabla 3. Espectro FTIR del extracto en diclorometano de hojas de Tecoma fulva subsp. arequipensis.

\begin{tabular}{cccl}
\hline $\mathbf{N}^{\circ}$ pico & í cm & $\begin{array}{c}\text { Vibración del } \\
\text { grupo funcional }\end{array}$ & \multicolumn{1}{c}{ Función orgánica } \\
\hline $\mathrm{A}$ & 3427 & $\mathrm{O}-\mathrm{H}, \mathrm{N}-\mathrm{H}$ & alcoholes, fenoles, ácidos y aminas \\
$\mathrm{B}$ & 2925 & $\mathrm{C}-\mathrm{H}$ & hidrocarburos saturados: alcanos \\
$\mathrm{C}$ & 2855 & $\mathrm{C}-\mathrm{H}$ & alcanos \\
$\mathrm{D}$ & 1699 & $\mathrm{C}=\mathrm{O}$ & cetonas $\alpha, \beta$ insaturadas, ácidos, ésteres \\
$\mathrm{E}$ & 1638 & $\mathrm{C}=\mathrm{C}$ & hidrocarburo insaturado: alquenos \\
$\mathrm{F}$ & 1514 & $\mathrm{C}=\mathrm{C}$ & hidrocarburo aromático \\
$\mathrm{G}$ & 1459 & $-\mathrm{CH}_{2-}$ & metilenos de alcanos \\
$\mathrm{H}$ & 1376 & $-\mathrm{CH}_{3}$ & metilos de alcanos \\
$\mathrm{I}$ & $1298-913$ & $\mathrm{C}-\mathrm{C}, \mathrm{C}-\mathrm{O}, \mathrm{C}-\mathrm{N}$ & huella dactilar: alcanos, éteres, aminas \\
\hline
\end{tabular}

La detección de los constituyentes químicos de los extractos en diclorometano de hojas y flores del T. fulva subsp. arequipensis, verificada por la técnica de FTIR, se encontró grupos funcionales característicos como: fenoles solamente en hojas, cuyas bandas de absorción son 1638 y $1514 \mathrm{~cm}-1$, mientras en las flores se encuentran alcoholes secundarios, en ambos extractos se encuentra el grupo carbonilo especialmente de cetonas $\alpha, \beta$ insaturadas, ácidos carboxílicos y ésteres, entre las bandas de absorción de 1699 y $1709 \mathrm{~cm}-1$, en las bandas de absorción 3427 y $3500 \mathrm{~cm}-1$ se detecta la presencia del grupo hidroxilo y amino; las estructuras orgánicas detectadas también se confirman con el análisis de los espectros obtenidos por GC-MS10-12.

\section{CONCLUSIONES}

Por extracción y caracterización física en la T. fulva subsp. arequipensisse se comprobó la presencia de componentes orgánicos volátiles solubles en solventes de baja polaridad.

Por cromatografía CG/EM, del extracto diclorometanico y etéreo se identificó terpenos y terpenoides.

Por cromatografía en columna y CG/EM del extracto etanólico de hojas en la fracción 1, se identificó $\gamma$-sitosterol, en la fracción 3 se identificó ácido hexadecanoico y en la fracción 4 se identificó 1,3-dihidro-3,3-dimetil-2H-indol-2-ona, siendo los componentes orgánicos de mayor abundancia en cada fracción.

En los espectros de FTIR de los extractos del T. fulva subsp. arequipensis, se confirma la presencia de alcoholes, compuestos carbonilicos, hidrocarburos aromáticos, fenoles y aminas. 


\section{AGRADECIMIENTO}

Agradecemos a la Universidad Nacional de San Agustín de Arequipa y UNSA Investiga, por su apoyo económico en la subvención del proyecto seleccionado "Estudio fisicoquímico, biológico y actividad antimicrobiana del aceite esencial de Tecoma fulva subsp. arequipensis (cahuato)".

\section{REFERENCIAS BIBLIOGRÁFICAS}

1. Taha KF, El-kashoury EA, Ezzat SM, Saleh NA. Antimicrobial and antioxidant activity of volatile constituents of the leaves of Tecoma smithii Will Wats. Global Journal of Medical Plant Research. Glob J Med Plant Res. 2016; 4(4): 16-22.

2. Anburaj G, Marimuthu M, Rajasudha V, Manikandan R. Phytochemical screening and GC-MS analysis of ethanolic extract of Tecoma stans (Family Bignoniaceae) Yellow Bell Flowers. J Pharmacogn Phytochem. 2016; 5(4): 172-175.

3. Sundas I, Zaheer K, Rizwana R, Anjum P. Evaluation of antibacterial activity of leaf extracts of Mansoa alliacea (Lam.) A: H. Gentry, Tecomaria capensis (Thumb) Space and Tecoma stans (L) Juss Ex. H. B. And K. J Biodivers Environ Sci. 2016; 9(1): 69-75.

4. Salem M, Gohar M, Camacho L, El Shanhorey N, Salem A. Antioxidant and antibacterial activities of leaves and branches extracts of Tecoma stans (L) Juss ex Kunth against nine species of pathogenic bacteria. Afr J Microbiol Res. 2013; 7(5): 418-426.

5. Dash S, Das C, Sahoo D, Sahoo A. Phytochemical composition anti-inflammatory and analgesic activities of Tecoma stans Linn. (Bignoniaceae). Nat Pharm. Technol. 2011; 1(2): 5-8.

6. Vidhya R, Fleming T. Assessment of the Cytotoxic Potential of Tecoma castanifolia (D. Don) Melch Flower Extract Again MCF-7 Cell Line. AJEthno. 2016; 3(1): 001-005.

7. Joselin J, Sarasabai T, Rajam A, Jeeva S. Phytochemical evaluation of bignoniaceae flowers. J Chem Pharm Res. 2013; 5(4): 106-111.

8. Sowjanya P, Srinivas P. Phytochemical investigation and antimicrobial properties of crude flower extract of Tecoma stans (L) juss. Ex. Kunth. Der Pharm Lett. 2017; 9(7): 24-34.

9. Sunitha K. Preliminary phytochemical investigations and immune modulatory screening of methanol extract of Tecoma stans leaves. J Chem Pharm Res. 2018; 10(7): 151-155.

10. Sandosh T, Paul M, Yesu J. Phytochemical analysis of Stylosanthes fructicosa using UVVIS, FTIR and GC-MS. Res J Chem Sci. 2013; 3(11):14-23.

11. Kumar K, Prasad D. Identification and comparison of biomolecules in medicinal plants of Tephrosia tinctoria and Atylosia albicans by using FTIR. Rom J Biophys. 2011; 21(1): 63-71.

12. Kalaichelvi K, Dhivya S. Screening of phyto constituents, UV-VIS spectrum and FTIR analysis of Micrococca mercurialis (L) Benth. Int J Herb Med. 2017; 5(6): 40-44.

13. Rodríguez M, Alcaraz M. Procedimiento para la extracción de aceites esenciales de plantas aromáticas. Centro de investigaciones biológicas del noreste S. C. La Paz, Baja California. México: Proyecto SAGARPA. CONACYT 126183; 2012. 
14. Rajamurugan R, Thirunavukkrasu C, Sakthivel V, Sivashanmugam M, Rghavan C. Phytochemical screening, antioxidant and antimicrobial activities of ethanolic extract of Tecoma stans flowers. Int J Pharma Bio Sci. 2013; 4(2): 124-130.

15. Ochoa K, Paredes L, Bejarano D, Silva R. Extraction characterization and evaluation of antibacterial activity of essential oil of Senecio graveolens Wedd (Wiskataya). Scientia agricultural. 2012; 3: 291-302.

16. Travati G, Cherice G, Sanchez E, Galhiane M. Essential oil composition of Aloysia gatissima from Brazil. J Essent Oil Res. 2009; 21: 325-326.

17. Essam F, Maytham H. Extraction and purification of terpenes from nutmeg (Myristica fragrans). J Al-Nahrain Univ. 2012; 15(3):151-160.

18. Wang Y, Li X, Jiang Q, Son H, Chen S. Guam D et al. GC-MS analysis of the volatile constituents in the leavers of 14 compositae plants. Molecules. 2018. 23(1): 166. doi: 10.3390/molecules23010166.

19. Peniche H. Identificación de compuestos leishmanicidas en el rizoma de Dorstenia contrajerva. [Tesis de Maestro en Ciencias]. Mérida, México. Centro de Investigación Científica de Yucatán; 2016. 